FORMATION Formation emploi

Revue française de sciences sociales

143 | Juillet-Septembre 2018

Le retour en formation : une vraie chance?

\title{
La « deuxième chance » : comparer des dispositifs diplômants de "ré-inclusion scolaire », en France et en Argentine
}

The "second chance" : comparing graduating "school re-including" plans, in

France and in Argentina

Die „zweite Chance“ : Wiedereinstieg mit Schulabschluss - Frankreich und

Argentinien im Vergleich

La "segunda oportunidad" : comparar dispositivos de "reingreso escolar" que otorgan certificados, en Francia y en Argentina

\section{Filippo Pirone}

\section{OpenEdition}

Journals

Édition électronique

URL : http://journals.openedition.org/formationemploi/6345

DOI : 10.4000/formationemploi.6345

ISSN : 2107-0946

Éditeur

La Documentation française

Édition imprimée

Date de publication : 20 novembre 2018

Pagination : 183-202

ISSN : 0759-6340

Référence électronique

Filippo Pirone, « La « deuxième chance » : comparer des dispositifs diplômants de « ré-inclusion scolaire », en France et en Argentine », Formation emploi [En ligne], 143 | Juillet-Septembre 2018, mis en ligne le 20 novembre 2020, consulté le 10 décembre 2020. URL : http://journals.openedition.org/ formationemploi/6345 ; DOI : https://doi.org/10.4000/formationemploi.6345 


\title{
La « deuxième chance » : comparer des dispositifs diplômants de « ré-inclusion scolaire », en France et en Argentine
}

\author{
FILIPPO PIRONE \\ Maître de conférences en sciences de l'éducation. Université de Bordeaux, École supérieure du \\ professorat et d'éducation d'Aquitaine, Laboratoire Cultures Education et Sociétés
}

Résumé

\begin{abstract}
La « deuxième chance » : comparer des dispositifs diplômants de « ré-inclusion scolaire », en France et en Argentine

Cet article se propose d'analyser les manières d'appréhender la " deuxième chance " au sein de l'école, en Argentine et en France. À travers l'étude et la comparaison de trois dispositifs diplômants de "ré-inclusion scolaire " d'anciens décrocheurs, nous montrons que, malgré les différences structurelles et contextuelles, les deux systèmes intègrent, en leur sein, les logiques contemporaines de l'inclusion scolaire, tout en les confinant à leurs marges. En effet, ces dispositifs ne peuvent pas suffire à renverser les mécanismes traditionnels d' "indifférence aux différences ", d'exclusion et de sélection scolaires et sociales.
\end{abstract}

Mots clés : système éducatif, abandon des études, mesure jeune, politique de l'éducation, enseignement secondaire, jeune en difficulté, projet d'établissement, comparaison internationale, France, Argentine

Abstract

The "second chance": comparing graduating "school re-including" plans, in France and in Argentina

This paper aims at analyzing the ways Argentinian school and French school deal with the "second chance". Through the study and the comparison of three "school re-including" plans for pupils who have previously dropped out of school, we show that, despite their structural and contextual differences, the two systems integrate contemporary ideas about inclusion, but marginalize them at their borders at the same time. In fact, these plans cannot be enough to overturn traditional mechanisms as the "indifference to differences", exclusion and school and social selection.

Keywords: education system, drop out, youth employment scheme, education policy, secondary education, young person in difficulty, school work plan, international comparison, France, Argentina

Journal of Economic Literature: I 21 ; $\mathbf{O} 57$

Traduction : Auteur 


\section{Introduction}

Au sein de nombreux systèmes éducatifs contemporains, on assiste à une montée d'un phénomène que l'on pourrait qualifier de "scolarisation des problèmes sociaux» (Smeyers $\&$ Depaepe, 2009). Il efface la séparation spatio-temporelle et symbolique traditionnelle entre l'école et le monde extérieur sur lequel elle est «ouverte» (Bernstein, 1997). Ainsi, l'école assume aujourd'hui une fonction de prise en charge des (nouveaux) besoins sociaux. Parmi ces derniers, à l'ère de la montée des logiques d'inclusion scolaire (Ebersold, 2009), celui de la remédiation du "décrochage " est considéré comme l'un des plus importants. Cela conduit les politiques d'éducation nationales et internationales à trouver des solutions permettant d'accorder aux « décrocheurs », ces élèves ayant abandonné précocement l'école, une " deuxième chance » de l'intégrer.

Nous nous proposons ici d'analyser et de comparer la façon dont les systèmes éducatifs français et argentin tentent de répondre à la question de la ré-inclusion scolaire (reingreso escolar), (cf. Poggi, 2009) d'une partie de leurs décrocheurs, en leur offrant une deuxième chance de reprendre leur formation scolaire et d'obtenir le diplôme de fin d'études secondaires. Nos analyses visent principalement à explorer ce qui nous semble constituer un paradoxe. Ainsi, alors que le système français accorde généralement beaucoup plus d'importance aux objectifs de poursuite généralisée des études qu'en Argentine, c'est en réalité au sein de ce pays fédéral, beaucoup plus qu'en France, que sont mis en place des dispositifs de formation "scolaire " dans lesquels l'insertion professionnelle n'est pas l'objectif central. Afin de mieux contextualiser ce paradoxe, il convient de rappeler quelques éléments factuels sur le décrochage, et les remédiations envisagées en France et en Argentine.

\section{- Un paradoxe}

Les taux de décrochage scolaire (abandono escolar) est moindre en France (environ $8,9 \%$ ) qu'en Argentine (environ 15,8 \%) ${ }^{1}$. En effet, si l'on compare les ratios respectifs des décrocheurs par rapport au nombre total des deux populations, le ratio argentin est presque trois fois plus élevé que le ratio français. Ce phénomène est lié à leurs pyramides des âges respectives, la population française, bien que plus nombreuse, étant aussi plus âgée ${ }^{2}$.

En outre, les enjeux liés à la ré-inclusion scolaire et au maintien à l'école des anciens décrocheurs ne paraissent pas les mêmes dans les deux pays. L'Argentine se focaliserait

1. Respectivement selon le Gouvernement français et le dernier rapport du CEA (2017) sur l'enseignement secondaire en Argentine, consultable sur http://www.ub.edu.ar/centros_de_estudio/cea/cea_numero_62. pdf (05/09/2017).

2. En Argentine, il existe environ 173800 décrocheurs, c'est-à-dire environ quatre personnes sur 1000, contre 98000 en France, soit environ 1,5 personnes sur 1000. 
ainsi avant tout sur des problèmes d' " ordre social " plus ou moins directement engendrés par le cumul du décrochage et du chômage. Ainsi, en Amérique latine, la lutte contre le décrochage scolaire contribue, notamment, au contrôle social de phénomènes tels que la déviance et/ou la délinquance juvénile (Tiramonti, 2011). La France, quant à elle, est aussi et surtout soucieuse des enjeux liés à la compétitivité économique mondiale. Dans ce contexte, on s'attendrait notamment à voir l'État français s'emparer du problème en proposant des solutions permettant aux décrocheurs de bénéficier d'une deuxième chance pour poursuivre leur formation scolaire. Pourtant, étonnamment, la France paraît miser principalement sur des dispositifs visant surtout l'accompagnement à l'insertion professionnelle («Ecoles de la Deuxième Chance »-E2C - ou les «Établissements Pour l'Insertion Dans l'Emploi »-EPIDE). Elle se focalise ainsi de facto davantage sur la lutte contre le chômage juvénile que sur l'objectif d'une prolongation généralisée des études, dévolu aux solutions " qui émergent du terrain ». En effet, malgré un article de la loi de 2003, qui accorde un "droit au retour en formation initiale sous statut scolaire " ${ }^{3}$, et le changement d'appellation de la "Mission Générale d'Insertion » en "Mission de Lutte contre le Décrochage Scolaire " ${ }^{4}$, les actions de l'État en termes de remédiation du décrochage scolaire demeurent majoritairement centrées sur l'offre d'une nouvelle chance de trouver rapidement un emploi (Melin, 2017)5. A contrario, à partir des années 2000, l'État fédéral argentin et la plupart de ses 24 juridictions se sont fortement investis dans la création de dispositifs de retour à (et de maintien dans) l'école. C'est par exemple le cas de la escuela de reingreso (" école de ré-inclusion »), dispositif créé en 2004 à Buenos Aires. Aussi, à partir de 2006, suite à l'entrée en vigueur des articles 13 et 14 de la ley de educación nacional (loi d'éducation nationale), qui permettent la création d'un sous-système éducatif de "gestion sociale », d'autres dispositifs de ré-inclusion tels que le bachillerato popular (" lycée populaire », dispositif que nous analysons ici) ont acquis le statut d'écoles secondaires à part entière, pouvant délivrer le diplôme de fin d'études.

Cela peut paraître encore plus paradoxal quand on sait, d'une part, l'emprise qu'exerce l'école sur l'ensemble de la société française, en lien avec l'étroite corrélation entre diplômes et trajectoires sociales (Dubet, Duru-Bellat \& Véretout, 2010) et, d'autre part, la moindre place accordée par le système scolaire argentin aux épreuves et aux pratiques de l'éducation formelle à l'école 6 .

3. Article 14 de la loi du 8 juillet 2013 d'« orientation et programmation pour la refondation de l'École de la République ".

4. La MLDS (anciennement MGI) est censée gérer les actions de lutte contre le décrochage scolaire.

5. Voir par exemple celles relevant des réseaux « FOrmation QUALification Emploi » (FOQUALE).

6. Contrairement au cas français, pour obtenir le diplôme argentin de fin d'études secondaires, les élèves ne doivent passer aucun examen final. 


\section{- Une approche comparative des « cas les plus contrastés » : les "dispositifs éducatifs"}

Afin d'explorer ce paradoxe, nous nous appuyons sur les résultats de deux enquêtes. Elles étudient trois dispositifs éducatifs diplômants ad hoc autour du besoin social de la ré-inclusion scolaire, c'est-à-dire des dispositifs de "retour à l'école » (Zaffran \& Vollet, 2016) pour des « anciens décrocheurs" (Pirone \& Rayou, 2012) ${ }^{7}$. La première enquête a été réalisée de 2009 à 2012, au sein d'un " micro-lycée " (ML) d'Ile-de-France, le J. Verne, dans le cadre de notre doctorat. Elle a été ensuite mobilisée pour cette comparaison avec la seconde enquête, effectuée de 2014 à 2017, dans le cadre d'un projet "Évaluation-orientation de la Coopération Scientifique-Sud » (Ecos-Sud : Rochex, Southwell \& Indarramendi, 2018) ${ }^{8}$, pour lequel nous avons observé deux dispositifs argentins : le premier à Buenos Aires, le «Bachillerato Popular» (BP) Caballito, et le second dans la province de Cordoba, le « Programa de Inclusión y Terminalidad» ("programme d'inclusion et de fin d'études »PIT), dans l'école secondaire Lavalle.

Une approche comparatiste en éducation peut certes permettre de mieux comprendre notre système (Novoa \& Yariv-Mashal, 2003). Cependant, la distance qui sépare les systèmes français et argentin peut potentiellement limiter sa portée explicative (d'où peutêtre la rareté d'enquêtes similaires). Il nous a donc semblé opportun ici de mobiliser la démarche comparative des " cas les plus contrastés ", développée par Clifford Geertz, autour d'une même catégorie analytique (la deuxième chance à l'école), sans éluder la diversité qui les traverse (Giraud, 2012).

Nous nous sommes ainsi focalisés sur l'analyse des "dispositifs éducatifs ", que nous appréhendons à un niveau méso-sociologique en tant que configurations intermédiaires (Bonnéry, 2009). Ils résultent de l'articulation entre, d'une part, une "mondialisation » des systèmes et des politiques éducatives et, d'autre part, la grande variété des pratiques des acteurs locaux auxquels ces politiques délèguent une partie de la gouvernance scolaire (Novoa, 2001). En effet, les dispositifs éducatifs se multiplient en tant que points de convergence de l'univers de l'action publique de "prévention à " ou de "remédiation par» l'école des problèmes sociaux et scolaires considérés comme les plus urgents (Barrère, 2013). Investis comme des laboratoires de l'innovation en éducation, ils ont en charge le traitement de la "diversité à l'école ", en offrant une deuxième chance d'inclusion à des populations d'élèves perçues comme biographiquement similaires (souvent parce qu'elles " posent problème » à l'école ordinaire), tout en les confinant aux marges du système.

\footnotetext{
7. Ainsi, nous ne nous intéresserons pas ici aux dispositifs non-diplômants, qu'il s'agisse de ceux visant à " prévenir le décrochage " (comme les " dispositifs relais »), ou de ceux visant à faciliter l'insertion professionnelle, comme les E2D ou les EPIDE.

8. Il s'agit d'un programme dans lequel des laboratoires français et d'Amérique latine hispanophone ont la possibilité de collaborer sur un projet de recherche comparative sur trois ans. C'est grâce à ce programme qu’a été financée la recherche dont est issue cet article.
} 
De ce fait, le ciblage actuel de ces populations repose sur des typologies construites sur la perception de la trajectoire de chaque élève (Rochex, 2010), soit (ex-) décrocheur, perturbateur, empêché-méritant...

\section{- Les trois critères de comparaison}

Ainsi, une approche de comparaison internationale exige plus que jamais de trouver des critères d'analyse mesurables. Nous en avons choisi trois, tous centrés sur le type de relation que chacun des trois dispositifs étudiés entretient avec sa périphérie :

1- La relation avec les instances en charge des politiques éducatives nationales ou territoriales:

Le dispositif a-t-il été conçu " par le haut », par une décision politique ? Ou bien s'agit-il du fruit d'une initiative " par le bas ", successivement reconnue et légitimée politiquement ? Dans quelle mesure son contexte de création influence-t-il son fonctionnement?

2 - La relation avec le contexte social dans lequel il se situe et ses objectifs :

Le dispositif vise-t-il à " redresser " la trajectoire scolaire d'anciens décrocheurs (comment ?), ou bien se constitue-t-il également comme une instance d'empowerment ${ }^{9}$ de compétences extra-scolaires multiples? Lesquelles, et avec quels objectifs ?

\section{3 - La relation avec le reste du système scolaire:}

Le dispositif a-t-il été conçu comme un modèle d'innovation (de quel type ?) capable d'irriguer le reste du système ? Ou son existence relève-t-elle uniquement d'une réponse à un problème éducatif contextuel ? Laquelle?

\section{Encadré 1. Méthodologie}

Au sein des trois dispositifs a été mené un travail ethnographique avec des observations et des entretiens semi-directifs (de trente minutes à une heure et demi) avec différents acteurs scolaires.

Nous avons tenté de comprendre le « programme institutionnel » de chaque dispositif, c'est-àdire, «le processus social qui transforme des valeurs et des principes en action et en subjectivité par le biais d'un travail professionnel spécifique et organisé » (Dubet, 2002, p. 24). Nous avons donc été attentifs aux éventuels écarts entre le fonctionnement réel des dispositifs et celui, formel, dicté par les textes institutionnels. Pendant nos observations (deux cours parmi ceux dispensés par les enseignants interviewés), nous nous sommes focalisés sur les types de « relations pédagogiques » qui se construisaient entre élèves et enseignants, à savoir plutôt « verticales » et " mediées » par les savoirs scolaires, ou alors « horizontales » et "personnalisées » (Bernstein, 2007b). Au cours des entretiens, nous avons tenté de saisir l'engagement des acteurs au sein des dispositifs, le sens qu'ils conféraient à leur expérience et la perception de leurs élèves.

9. Entendu comme l'octroi de " pouvoirs " aux individus. 
Pour le micro-lycée (ML) J. Verne, nous avons interviewé un de ses fondateurs et enseignant d'histoire-géographie, ainsi qu'une enseignante en anglais. Le premier, enseignant agrégé de 40 ans, a fondé les deux premiers ML français et a longtemps travaillé pour les missions « innovation » de la Direction générale de l'enseignement scolaire. La seconde est une enseignante certifiée de 40 ans, ayant obtenu un temps plein au ML, où elle peut expérimenter l'enseignement de certaines techniques de relaxation de yoga, acquises pendant son séjour en Inde, en lien avec les enseignements de langue vivante.

Pour les terrains argentins, nous avons interviewé la directrice de l'école Lavalle, enseignante en psychopédagogie, le coordinateur du PIT (programme d'inclusion et de fin d'études) Lavalle et deux enseignants du BP (Bachillerato popular) Caballito en philosophie politique : Estéban et Pablo. La directrice de l'école Lavalle, femme de 50 ans, diplômée d'une licence en psychologie, collabore de manière étroite, depuis trois ans, avec le ministre de l'Éducation de la juridiction de Cordoba et deux autres proviseurs, autour de la conception de dispositifs représentant une alternative au redoublement. Le coordinateur du PIT est un enseignant de 50 ans en Éducation physique et sportive (licencié en STAPS - Sciences et techniques des activités physiques et sportives). Il a été nommé coordinateur par la directrice, qui paraît largement s'appuyer sur sa longue expérience professionnelle. Les deux enseignants du BP ont tous deux 35 ans et sont diplômés d'un master en sciences politiques. Estaban représente le BP Caballito au sein du Réseau des Bachilleratos Populares et, tout comme Pablo, il a participé à la création de la structure. Tout en exerçant dans d'autres classes du système éducatif, pour ces deux enseignants, comme pour d'autres de leurs collègues, le choix d'enseigner dans cette structure est un signe d'engagement politique, mais aussi une possibilité de se former et d'enrichir leur CV. II s'agit pour eux de pouvoir postuler sur des emplois plus stables dans des écoles classiques, afin de stabiliser une situation professionnelle précaire.

Enfin, nous avons recueilli des données sur les élèves : nombre, origines, trajectoires. Pour le ML, nous avons étudié les résultats scolaires et les trajectoires de deux promotions. Pour les dispositifs argentins, nous avons étudié les archives administratives.

L'article s'articule en trois parties, suivant l'ordre des trois critères d'analyse précisés cidessus axés sur l'étude et la comparaison des relations que chacun des trois dispositifs entretient avec sa périphérie. La première partie est centrée sur l'analyse de leurs relations avec les instances en charge des politiques éducatives nationales ou territoriales. Cette analyse permet notamment d'introduire une présentation générale des dispositifs. Dans la deuxième partie, nous analysons leur relation au contexte social. Elle permet notamment de comprendre leurs objectifs éducatifs sous-jacents. Enfin, dans une troisième partie, nous illustrons leurs relations avec le reste du système éducatif, afin d'éclairer le rôle que chaque dispositif semble jouer dans le processus d'innovation scolaire. 


\section{Des dispositifs imposés par le haut ou impulsés par le bas?}

Nous analysons ici la relation que les trois dispositifs entretiennent avec les instances en charge des politiques éducatives nationales ou territoriales. Dans une époque caractérisée par une décentralisation de la gouvernance scolaire du niveau macro vers le micro, ce type de comparaison nous permet de rendre compte des contextes qui ont présidé à la création des trois dispositifs et ont permis leur mise en place. Dans les sous-parties ci-dessous, nous analysons respectivement le BP, le PIT et le ML, pour ensuite synthétiser les analyses.

\subsection{Le « bachillerato popular » : une victoire des mouvements populaires précarisés par la crise de 2001}

En Argentine, le développement des politiques néolibérales au cours des années 1990, ayant abouti, en 2001, à l'une des crises économiques majeures de l'histoire du pays, a largement contribué à un double phénomène. Du côté de l'institution scolaire, l'État et les juridictions ont progressivement désinvesti l'école publique, au profit de l'école privée. Au niveau social, l'Argentine a enregistré une hausse considérable du chômage. De nombreuses usines et entreprises ayant fait faillite ont été réinvesties et revitalisées par des collectifs de travailleurs, au travers de pratiques d'autogestion. C'est précisément au sein de ces deux mouvements (chômeurs et travailleurs en autogestion) qu'est né le BP, une des victoires concédées par l'État aux mouvements sociaux en échange de la paix sociale (Torres Molina, 2012).

Les premiers BP ont vu le jour à Buenos Aires, en 2004, notamment grâce au « Movimeiento Popular La Dignidad" (MPLD) ${ }^{10}$, et on en dénombre environ 86 dans tout le pays, bien qu'environ $95 \%$ d'entre eux sont situés à Buenos Aires ou dans sa région. En 2014, ils revendiquaient l'accueil d'environ 2300 élèves $^{11}$ :

"Certains BP se font financer par le ministère : les profs perçoivent des salaires (...). Ici, on est tous bénévoles, on veut garder notre autonomie». (Pablo)

Créé en 2009, Caballito fait partie des 40 BP reconnus officiellement par l'État pour l'octroi du diplôme de fin d'études secondaires. Comme la plupart d'entre eux, cette structure refuse elle aussi d'être financée par le ministère de l'Éducation. Il s'agit d'éviter que l'administration publique n'interfère dans le fonctionnement interne. Ainsi, comme d'autres BP, Caballito s'autofinance lui aussi grâce au commerce solidaire du MPLD, ses enseignants étant tous bénévoles.

10. «Mouvement Populaire La Dignité " : il s'agit d'un des mouvements nés à la même époque à partir de celui des "Piqueteros", dont le slogan était : "Pain, travail, dignité et changement social».

11. Voir les publications en ligne du "Grupo de Estudios sobre Movimientos Sociales y Educacion Popular " (GEMSEP) : http://gemsep.blogspot.fr/p/publicaciones.html (consulté le 05/09/2017). 


\subsection{Le PIT : un « bouche-trou » pour respecter l'obligation scolaire prolongée}

Le PIT ${ }^{12}$, quant à lui, est créé en 2010, dans la juridiction de Cordoba, afin que la loi d'éducation nationale de 2006, qui relève l'obligation scolaire à l'âge de18 ans, puisse s’appliquer. Le PIT observé a d'ailleurs été le premier site à ouvrir.

Le 38 PIT existants ont été conçus en fonction des directives institutionnelles précises émanant du ministère de l'Éducation de la province de Cordoba. Nous en présenterons quelques-unes plus loin.

"Le ministre de l'Education de Cordoba pense que le PIT est un bouche-trou qui ne devrait même pas exister ». (Le coordinateur du PIT Lavalle)

Aux dires du coordinateur et de la directrice du site observé, le ministre dévaloriserait totalement le PIT, en le considérant comme une sorte de solution-tampon censée disparaître lorsque le respect de l'obligation scolaire jusqu'à l'âge de 18 ans sera effectif.

\subsection{Le micro-lycée ou l'institutionnalisation d'une innovation locale}

Dans un processus très différent de celui ayant présidé à la naissance du PIT, l'ouverture du premier ML français a été officialisée en 2000, grâce à une équipe d'enseignants très mobilisés d'un lycée, qui ont négocié pendant quatre ans avec les instances décisionnelles de l'Éducation nationale :

"En 2000, lorsque Jack Lang arrive au ministère de Grenelle (...), il cherche tout de suite des objets pédagogiques qui puissent être mis dans la vitrine. (...) Nous avions entretemps enterré ce projet, au bout de quatre ans, on en avait assez, on en avait marre de courir après, et donc si (...) Jack Lang ne nous avait pas recontactés, (...) le ML n'aurait jamais vu le jour. » (Co-fondateur du ML J. Verne et du premier ML)

Aujourd'hui, il existe une douzaine de ML (deuxième micro-lycée, le ML J. Verne a été créé en 2008). Avec d'autres structures similaires, il fait partie de la Fédération des établissements scolaires publics innovants (FESPI, créée en 2005). Ses missions sont consultatives au sein du ministère de l'Éducation nationale. Le fondateur des deux premiers ML a contribué à une labélisation nationale des ML qui, depuis la loi de 2013, font désormais partie du réseau des «Structures de Retour à l'Ecole » $(\mathrm{SRE}){ }^{13}$.

12. Les données et les informations générales sur le PIT s’appuient sur les travaux de Vanella \& Maldonado (2013).

13. Valérie Melin (op. cit.) montre bien que le programme des SRE, qui ont été fondées suite à la loi de 2013, sous le label " micro-lycée ", s'éloigne totalement de celui des premières structures des années 2000. En effet, les nouveaux ML se distingueraient à peine des nombreux autres dispositifs d'État de remédiation du décrochage scolaire, centrés sur l'insertion professionnelle. 


\subsection{Des dispositifs impulsés par le haut ou par le bas}

Selon leur modalité de création, des dispositifs bottom-up peuvent exister qui, en partant du local et/ou du " bas » du système (comme le BP et le ML) parviennent, au travers des luttes et/ou des négociations, à se faire reconnaître par l'institution, à perdurer, voire à reproduire leur modèle sur le territoire (ou en tout cas sur une partie de ce dernier). Leur pérennisation et leur essaimage sont liés au fait qu’ils sont parvenus à combler un vide relatif laissé par les États en matière de ré-inclusion scolaire. Le PIT, quant à lui, est plutôt un dispositif top-down, puisqu'il a été imposé localement depuis les hautes sphères institutionnelles (Cros, 2001) ${ }^{14}$. En effet, sa fonction de ré-inclusion scolaire paraît périphérique par rapport à celle de respect effectif de l'élévation de l'âge de l'obligation scolaire en Argentine.

Malgré leurs différences, les trois dispositifs bénéficient d'une autonomie pédagogique et financière supplémentaire par rapport à la scolarisation classique ${ }^{15}$. Par exemple, les trois dispositifs peuvent non seulement recruter en interne les membres de leur équipe pédagogique, mais aussi sélectionner leurs nouveaux élèves.

\section{Principes implicites et objectifs des trois dispositifs}

Le deuxième critère d'analyse concerne la relation de chaque dispositif avec son contexte. Si les écoles de la République française et d'Argentine se sont développées sur des bases d'indifférence à la différence, voire d'exclusion de l'hétérogénéité et de la diversité liée au "non-scolaire » (Dussel, 2004), quels sont les principes implicites des dispositifs et leurs objectifs réels ? Dans les sous-parties ci-dessous, nous analysons respectivement le BP, le PIT et le ML, pour ensuite synthétiser les analyses.

\footnotetext{
14. Il s'agit d'une catégorisation utilisée par l'auteure, en référence aux innovations en éducation. Au cours de notre thèse, nous avons constaté que les innovations top-down (du haut vers le bas) disposent généralement d'importants moyens de réalisation immédiats, mais que leur pérennisation est davantage fragilisée par les évolutions électorales que les innovations bottom-up (du bas vers le haut). En effet, bien que celles-ci peinent à se faire reconnaître institutionnellement et à obtenir des moyens pour leur fonctionnement, elles jouissent généralement d'une grande autonomie vis-à-vis des sphères politiques.

15. En France, par exemple, l'article 34 de « loi d'orientation et de programmation pour l'avenir de l'école ", de 2005, permet de concevoir et de mettre en place des dispositifs expérimentaux à caractère innovant qui bénéficient d'un statut dérogatoire.
} 


\subsection{Le « bachillerato popular » : à l'école de l'engagement politique}

En accueillant des anciens décrocheurs de tous âges, le BP ne naît pas seulement d'un souci de les ré-inclure dans un système secondaire qu'ils ont dû abandonner trop précocement. Il est aussi et surtout censé leur offrir une éducation leur permettant de s'émanciper socialement, en stimulant leur sens critique et leur engagement politique.

Le lien très fort entre le BP et la sphère de l'engagement politique et social est notamment visible dans le fait que les différents BP créés sont étroitement articulés à des organisations sociales (centres culturels, collectifs sociaux, etc.) dont ils dépendent administrativement et financièrement :

"Lorsqu'ils viennent s'inscrire ici, on essaie toujours de les faire participer à la vie de notre association (...), de les impliquer politiquement. (...) Beaucoup d'élèves et profs ici sont aussi des militants ». (Estéban)

Ce lien est également visible dans leur organisation curriculaire. En effet, dans l'offre de cours coexistent des disciplines plus classiques (mathématiques, économie) et d'autres inédites, comme " analyse de la conjoncture ", " organisation et participation populaire " ou « autogestion».

\subsection{Le PIT : entre « maintien scolaire » et transition vers l'emploi}

Le PIT, quant à lui, accueille des élèves âgés de 14 à 17 ans, majoritairement issus des milieux populaires, vivant ou ayant vécu des difficultés socio-économiques qui les ont éloignés de l'école. Beaucoup d'entre eux sont entretemps entrés dans la vie active et certains ont eu des enfants.

Une partie de l'offre pédagogique du PIT se focalise sur la préparation à l'entrée dans le monde du travail. En effet, au sein du PIT Lavalle, comme dans beaucoup d'autres, les élèves suivent des cours de "formation pour la vie et le travail » et de "formation professionnelle ", encadrés par des intervenants issus du monde de l'entreprise et de l'orientation professionnelle.

Malgré les recommandations ministérielles visant à accroître les efforts afin que les élèves puissent compléter leur cycle secondaire, le taux d'abandon au sein du PIT étudié est d'environ un élève sur deux :

"Pour beaucoup de jeunes, il est trop difficile d'être à la fois travailleur et élève (...). A un moment donné, il y en a qui finissent par craquer ». (Coordinateur du PIT Lavalle)

Ceux qui parviennent à s'accrocher au dispositif sont censés se présenter devant un jury en fin d'année, afin de valider un oral devant leur permettre de passer dans la classe supérieure. En réalité, seulement un élève sur quatre environ s'y présente, ce qui n'empêche apparemment pas tous les " déserteurs " jugés " assidus " par leurs enseignants de valider l'année. Même s'ils ne semblent pas vouloir s'attarder sur le sujet, le coordinateur et la 
directrice du site nous ont confié qu'ils préferent ne pas sanctionner ces pratiques, plutôt que risquer de "perdre " l'année suivante des élèves pouvant ne pas supporter l' "échec » ("fracaso »).

\subsection{Le micro-lycée : le baccalauréat comme objectif ultime}

Le ML, quant à lui, a été créé pour aider des lycéens de 16 à 25 ans, sortis du système scolaire pendant au moins six mois, à obtenir le baccalauréat.

Situé dans une banlieue populaire proche de Paris, le ML J. Verne accueille une population d'élèves ayant pour la plupart 19-20 ans, ne résidant pas tous à proximité de la structure, et issus de milieux sociaux très variés (avec une égale répartition entre les classes populaires, moyennes et moyennes-supérieures).

Bien que l'équipe pédagogique soit focalisée sur l'objectif lié à l'assiduité scolaire, tous les ans, à partir de la veille des vacances de Noël, elle assiste à une recrudescence de l'absentéisme et des retards.

Les taux d'abandons, au cours d'une année scolaire, oscillent entre 10 et $20 \%$, et $65 \%$ environ des élèves de Terminale obtiennent le baccalauréat chaque année :

Enquêteur : "Tu as vu des effets 'consommation du ML' de la part des élèves ici ?"

"(...) Oui, mais je m'en fous. Même sy'a des profs que ça dérange (rires). (...) Je veux dire, moi, si un élève fait son chemin en nous consommant avec utilité, voire utilitarisme, et qui réussit sa trajectoire, après tout, c'est bien pour lui!». (Co-fondateur du ML J. Verne)

Malgré la grogne de certains enseignants qui ne se sentent pas à l'aise face à des élèves ne participant pas aux efforts collectifs de co-construction de la structure, tout en réussissant scolairement, le fonctionnement de la structure paraît bien se centrer sur son objectif ultime, à savoir faire obtenir le baccalauréat au plus grand nombre d'élèves inscrits, ce qui fait aussi la fierté de certains enseignants :

"Tous les ans, environ 75-80\% de nos jeunes, c'est-à-dire des élèves qui sans le ML n'auraient très probablement pas pu poursuivre leur scolarité, arrivent à valider leur année scolaire. (...) Pour nous, c'est une fierté"». (Enseignante d'anglais au ML J. Verne)

\subsection{Des dispositifs centrés sur du « scolaire », du « professionnel » ou du « citoyen »}

Ainsi, selon leur programme institutionnel, chaque dispositif semble mettre en avant un ou plusieurs types de connaissances et de compétences.

En affichant comme objectif principal l'accompagnement de ses élèves vers le baccalauréat, le ML apparaît, par exemple, principalement centré sur des enjeux et des contenus «scolaires". 
Si cette focalisation sur le scolaire est bien présente aussi au sein du PIT, ce dispositif propose également un travail de préparation à l'entrée dans le monde du travail, qui occupe une large part de son offre pédagogique. Ainsi, son programme institutionnel paraît se déployer parallèlement autour d'enjeux « scolaires » et " professionnels » (c'est-à-dire liés à des perspectives d'insertion dans le monde du travail).

$\mathrm{Au} \mathrm{BP}$, en revanche, la dimension scolaire semble bien plus marginalisée, la plupart des disciplines proposées étant inédites et paraissant viser davantage la formation d'un citoyen émancipé, critique et « engagé » que d'un «bon élève ». Ainsi, le programme institutionnel du BP semble essentiellement focalisé sur des enjeux de " citoyenneté ».

Les deux dispositifs argentins paraissent ainsi plus enclins que le ML à embrasser les logiques de l'ouverture de l'école à des instances et à des acteurs provenant de l'univers social de l'associatif et du territoire. Cela contribue à une montée des pédagogies "invisibles» (Bernstein, 2007a) déployées au travers de contenus, de pratiques et d'outils hybrides entre le monde scolaire et l'univers du non-scolaire, et centrées moins sur l'objectif d'acquisition de connaissances que sur celui d'acquisition de compétences multiples (Rey, 2014).

\section{Des relations « extensives » ou « intensives » avec le reste du système scolaire »?}

Le troisième critère d'analyse concerne la relation que chaque dispositif entretient avec le reste du système scolaire dans lequel il se situe.

Dans les deux pays, comme au sein de beaucoup d'autres, s'est instauré et stabilisé un mode d'enseignement vertical, simultané et dirigé vers une collectivité d'apprenants regroupés selon leur niveau d'âge (la classe) et qui suivent tous la même progression linéaire dans les curricula. Comme il revient de plus en plus aux acteurs locaux d'innover à l'école et de trouver de nouvelles solutions pouvant alimenter l'ensemble du système éducatif, dans quelle mesure les trois dispositifs sont-ils novateurs ? Comme pour les deux parties précédentes, dans les sous-parties ci-dessous, nous analysons respectivement le BP, le PIT et le $\mathrm{ML}$, pour ensuite en réaliser une synthèse.

\subsection{Le « bachillerato popular » : une école «à part (entière) »}

Les $\mathrm{BP}$ sont des établissements d'enseignement secondaire à part entière. Leur programme institutionnel ne met pas seulement en avant un modèle alternatif d'école, celui d'une école réellement démocratique, émancipatrice et inclusive, mais il propose également de réformer profondément le paysage politique et social argentin. Cela est lié au fait que le fonctionnement du BP repose sur la tradition de l'éducation populaire, les théories de 
Paulo Freire et les principes de l'autogestion ${ }^{16}$. Pour cela, la quasi-totalité des décisions autour de la vie collective et pédagogique du BP Caballito se prennent lors des assemblées générales qui se tiennent toutes les trois semaines et qui se composent de l'ensemble des élèves et des enseignants.

Quant aux réunions de l'équipe pédagogique, elles se déroulent les week-ends et/ou pendant des jours fériés, et les décisions y sont prises par consensus. Les enseignants y discutent notamment des passages des élèves d'une année à l'autre, des affectations, de l'obtention ou non du diplôme de fin d'études :

"Nous sommes obligés de nous inventer des notes pour délivrer le diplôme d'État aux élèves qui complètent le cycle». (Pablo)

Les appréciations sur les élèves s'appuient notamment, comme au sein du PIT, sur l'assiduité et la participation active aux activités du dispositif. En ce sens, pour l'équipe pédagogique, le recours à une note "sommative négociée " paraît représenter un compromis entre le choix initial de ne pas noter et les contraintes administratives liées au système éducatif fédéral.

La distance du BP par rapport au modèle classique d'école apparaît évidente lorsque l'on étudie la configuration et l'ambiance du BP Caballito, situé dans un quartier populaire de Buenos Aires. En effet, les locaux de Caballito sont implantés dans le stade d'une équipe professionnelle de football, dans l'enceinte délimitant les tribunes. Cet établissement scolaire accueillant environ 40 élèves par an se présente comme une petite enclave comprenant trois salles de cours séparées par une sorte de sas où se trouvent un baby-foot et une bibliothèque de quelques dizaines de livres.

L'ambiance de l'établissement s'inscrit fortement dans l'informel et la convivialité : nombre d'élèves et d'enseignants (résidant dans le quartier pour la plupart) y entrent avec leurs vélos, se font la bise en arrivant, se tutoient et partagent du maté pendant les cours ${ }^{17}$.

"Une bonne partie d'entre nous se connaît bien même en dehors des cours (...). Ça nous arrive de nous retrouver, profs et élèves confondus, le weekend, notamment autour des activités de l'association ». (Estéban)

16. Voir "Pedagogia de la rebeldia. Bachilleratos Populares. MPLD", une sorte de manifeste présentant les principales lignes de la philosophie et du fonctionnement du dispositif. Consultable sur : https://issuu.com/ gabynete/docs/bachilleratos_populares_pedagog_a_ (05/09/2017).

17. Il s'agit d'une boisson traditionnelle sud-américaine préparée en infusant des feuilles de yerba mate qu'on boit collectivement dans une coupe spéciale, à l'aide d'une paille en métal. Une fois la coupe terminée, chaque buveur à son tour la remplit à nouveau avec de l'eau très chaude contenue dans un grand thermos, avant de la passer à son voisin. C’est une pratique à la fois très habituelle et conviviale en Argentine et dans d'autres pays limitrophes. 
Comme beaucoup d'autres, ce BP est organisé en trois niveaux de classe $\left(1^{\text {ère }}, 2^{\text {ème }}\right.$ et $3^{\text {ème }}$ année), en raccourcissant de fait le cycle secondaire ${ }^{18}$.

\subsection{Le PIT : une « école-chorizo » pour « aimer les élèves »}

Les PIT sont, quant à eux, tous hébergés dans une école secondaire (un IPEM : Institut Provincial de l'Enseignement Moyen), mais ils gardent leur autonomie et un statut dérogatoire leur permettant de disposer de moyens exceptionnels. Parmi ceux-là :

- Des groupes de 20 élèves maximum ;

- Des journées de quatre heures de cours maximum ;

- Un cycle secondaire complet de seulement quatre ans (contre les six prévus normalement dans la juridiction);

- L'instauration d'un climat de dialogue et d'un traitement personnalisé des élèves ;

- L’aménagement des espaces de vie pour les élèves ${ }^{19}$;

- La présence (entre autres) d'un coordinateur pédagogique travaillant étroitement avec la direction de l'IPEM, d'un psychopédagogue et d'enseignants pouvant jouer le rôle de tuteurs personnalisés auprès des élèves ;

À la simultanéité classique de l'espace-temps " classe » sont préférés des « espaces éducatifs ", où l'élève peut acquérir, individuellement ou en petits groupes, des savoirs différents de ceux acquis par certains de ses camarades dans la même configuration spatio-temporelle ${ }^{20}$.

Le PIT Lavalle est situé dans une banlieue populaire de la ville de Cordoba. C'est le plus grand de la province, avec l'accueil d'environ 120 élèves par an. La directrice de l'IPEM et le coordinateur du PIT l'appellent souvent l' " école-chorizo ». En effet, de la même manière que ce type de saucisson est contenu dans un intestin, les murs de Lavalle contiennent le PIT (et un autre dispositif annexe). Le matin et en début d'après-midi, Lavalle est une école classique (avec une spécialisation " tourisme »). Le soir, elle se transforme en PIT, avec une spécialisation sciences économiques et sociales :

"Le PIT propose une vraie nouvelle solution éducative et a un programme curriculaire défini!». (Coordinateur du PIT Lavalle)

18. À Buenos Aires, le cycle habituel de l'école secondaire est de cinq ans. En outre, au BP Caballito, les heures de cours hebdomadaires (se déroulant le soir, pour permettre la présence des travailleurs) sont réduites de moitié par rapport à celles d'un lycée classique (une quinzaine contre une trentaine).

19. Par exemple, le PIT Lavalle est le premier dispositif de l'histoire de la province à accepter les enfants des élèves-parents. Aux dires de la directrice, certains enseignants se transforment en «tontons " ou " tatas ». 20. Il s'agit d'espaces-temps similaires à ce que nous appelons une "étude surveillée ", où un seul enseignant encadre l'activité d'apprentissage de sous-groupes d'élèves de différents niveaux, travaillant sur des matières diverses. 
Les coordinateurs et la directrice paraissent investir le dispositif comme une véritable alternative aux structures secondaires classique :

"(...) Il permet ce qui serait impossible à l'école classique : pouvoir comprendre à 360 degrés les jeunes, les aimer, pouvoir les entraîner à savoir se valoriser lors d'un entretien d'embauche, leur laisser une heure toutes les semaines pour échanger sur leurs problèmes (...) et travailler sur l'empathie, l'auto-estime, l'écoute réciproque (...) pour pouvoir 'positiver' toujours avec des jeunes déjà lourds d'une vie difficile ». (Directrice de l'IPEM Lavalle)

\subsection{Le micro-lycée : une école pour « réparer des identités cassées »}

Dans la "charte des ML » ${ }^{21}$, on trouve certains éléments constitutifs du dispositif, comme "Favoriser des approches diversifiées des savoirs: inter et transdisciplinarité, pédagogie différenciée. (...) Les tâches, élaborées en équipe et définies dans le cadre d'un cahier des charges, sont multiples et relativement polyvalentes : enseignement, accompagnement éducatif, vie scolaire, administration, relations extérieures".

Comme au PIT Lavalle, le ML J. Verne se situe dans une aile isolée d'un lycée polyvalent dont il dépend officiellement, mais avec qui il n'entretient de facto aucune relation. Cela est le signe d'un fort degré d'autonomie (non seulement symbolique) du dispositif visà-vis du système éducatif classique. En effet, le ML n'opère pas dans la perspective de la ré-inclusion de ses élèves au sein de ce dernier, mais plutôt, plus généralement, au sein $\mathrm{du}$ « monde scolaire » tout court (dont le ML fait désormais pleinement partie, bien que demeurant à ses marges).

La structure observée comporte une "salle commune ", son véritable cœur, lieu à la fois de vie et de convivialité pour les enseignants et les élèves (qui se tutoient), d'apprentissage, de réunion, ou encore de vie administrative (en arrivant, les élèves signalent leur présence dans un " cahier du bonjour»).

On compte 10 enseignants et 90 élèves environ. Dans une structure sans hiérarchie, les enseignants se partagent l'intégralité des tâches (y compris la surveillance et l'administration).

Les relations pédagogiques de la structure se centrent sur le principe de la confiance et sont caractérisées par un travail de "réparation des identités cassées" (aux dires du fondateur) d'élèves qui, comme ceux du PIT $^{22}$, ont, pour la plupart, décroché à cause d'expériences passées problématiques avec l'institution scolaire et avec des enseignants " cassants ", c'est-

21. Consultable sur : http://www.fespi.fr/wp-content/uploads/2015/03/Charte_ML_-_ver_def_ au_21.04.09.pdf (05/09/2017).

22. Les personnels du PIT semblent croire fortement à ce que de nombreux élèves leur disent régulièrement, à savoir que les raisons de leurs échecs seraient liées aux « mauvaises relations " qu'ils disent avoir eues avec leurs enseignants. 
à-dire qui n'ont pas pu/su, à leurs yeux, faire preuve de respect à l'égard de leur identité de "jeunes» (Pirone, 2015) ${ }^{23}$ :

"L'enseignante demande aux élèves de repérer les mots les plus importants dans le titre du texte qu'ils ont sous les yeux. Certains élèves lisent les mots à voix haute. Elle les félicite avec insistance. (...) Elle félicite grandement un élève pour avoir repéré dans le texte le mot qu'elle avait demandé de trouver. (...) Pendant le cours, la phrase "Congratulations!" ("Félicitations!") est prononcée par l'enseignante à maintes reprises (à chaque fois qu'un élève accomplit correctement une petite tâche). (Notes prises pendant le cours de l'enseignante d'anglais du ML J. Verne)

Ce type de pédagogie est quasiment dépourvu de sanctions et d'évaluations sommatives, répondant en cela à des élèves désireux d'être reconnus comme des " jeune-adultes ", requalifiés symboliquement et rassurés sur leur possibilité de se " racheter " scolairement. Ainsi, les enseignants n'ont de cesse de féliciter leurs élèves à chaque occasion où ils paraissent accomplir leur métier d'élèves.

Parmi les moyens exceptionnels dont il dispose, le ML bénéficie, comme le PIT, de classes à effectifs réduits :

"Je travaille pour démultiplier les structures de type ML à l'échelle nationale .» (Co-fondateur du ML J. Verne)

En partageant le programme de la $\mathrm{FESPI}^{24}$, ce dispositif s'érige en modèle pouvant permettre à l'école française d'innover et de contribuer à son " évolution démocratique ».

\subsection{Des dispositifs pour innover de façon « extensive » ou « intensive »}

Ainsi, le troisième critère de comparaison permet de révéler si la volonté institutionnelle autour du programme de ces dispositifs est bien de permettre au système d'innover " en profondeur » (" intensivement »). Comme nous l'avons vu précédemment, c'est le cas du ML et du BP qui, en ne se contentant pas seulement de répondre à un besoin social ponctuel (" extensivement », cf. Terigi, 2013) ${ }^{25}$, s'érigent en modèles alternatifs capables de renouveler le système éducatif (voire la société, dans le cas du BP).

L'exemple du PIT est, quant à lui, plus difficile à catégoriser. En effet, la portée de ce dispositif est intensive aux yeux des acteurs observés au sein de Lavalle, qui le considèrent comme un meilleur modèle éducatif que l'école traditionnelle. Toutefois, pour le ministre

23. Au cours de notre enquête au sein du ML, plusieurs élèves interrogés estimaient que leurs anciens enseignants ne se souciaient guère de leur "cas", de leurs problèmes personnels et les traitaient comme des "numéros", voire faisaient preuve d'un certain mépris vis-à-vis d'eux.

24. Voir : http://www.fespi.fr/la-fespi/ce-que-nous-souhaitons/ (consulté le 05/09/2017).

25. Dans sa classification des innovations en éducation, l'auteure distingue les innovations "intensives", c'est-à-dire très contextuelles et qui visent une innovation "profonde ", des innovations " extensives ", à savoir très diffuses sur le territoire et visant une innovation marginale du système " traditionnel ». 
de l'Éducation de Cordoba, il s'agit plutôt d'une solution temporaire censée disparaître une fois la question du strict respect de l'obligation scolaire résolue, ce qui ne nous paraît pas aussi simple à réaliser dans le court terme.

Malgré ces différences, les trois dispositifs semblent tous fonctionner autour de la tendance actuelle de l'école à se penser comme une "institution de vie " (Acosta, 2009) accordant une attention particulière aux individus, notamment ceux qui occupent les positions les plus dominées et qui sont en quête d'une reconnaissance et d'une valorisation de leurs identités (Taylor, 1992).

\section{Conclusion}

Comme nous l'avons vu, les dispositifs étudiés paraissent tous épouser les traits du mouvement contemporain de reconfiguration des scolarités visant à inclure le plus grand nombre d'élèves dans l'école. Ces objectifs se déploient à travers l'horizontalité exacerbée de relations pédagogiques de plus en plus personnalisées et de moins en moins médiées et qui, dans un effort collectif de (re-)valorisation des identités d'élèves ayant préalablement rompu avec l'école, se centrent sur les principes de la confiance réciproque. En ce sens, il ne s'agit pas seulement de dispositifs donnant une deuxième chance d'être inclus scolairement, mais aussi et surtout de se « maintenir » dans l'école.

Ainsi, les objectifs, les contenus et les moyens d'action strictement liés aux savoirs scolaires y apparaissent comme de moins en moins centraux, puisque d'autres types de connaissances et de compétences peuvent être mis en avant. On assiste ainsi, notamment au sein des dispositifs observés en Argentine, à des scolarisations à "basse intensité " (Kessler, 2006), où les apprentissages et les enjeux proprement scolaires sont assez faiblement investis. Si pour le ML, cela paraît beaucoup moins vrai, il faut rappeler qu'en France, les E2C (Écoles de la deuxième chance), c'est-à-dire des dispositifs non diplômants visant principalement une insertion professionnelle rapide, sont au moins dix fois plus nombreuses ${ }^{26}$ que les ML (micro-lycées). En effet, malgré l'intérêt que suscite le modèle au sein des sphères décisionnelles, depuis plus de sept ans ${ }^{27}$, le ML ne représente qu'une exception dans le paysage des dispositifs de remédiation du décrochage scolaire.

Dans une configuration socio-historique où l'on accorde une grande importance à la question de la deuxième chance à l'école, entendue comme la possibilité offerte aux décrocheurs de réintégrer le système scolaire pour parvenir à compléter le cycle d'études secondaires, les systèmes éducatifs argentin et français paraissent réagir de manière inverse.

26. On en compte 118.

27. La volonté institutionnelle d'essaimage du « modèle ML " avait déjà été exprimée par la circulaire ministérielle de la rentrée scolaire 2010, où était annoncée l'intention de créer un ML dans chaque Académie. Bien que l'appréciation positive sur ce dispositif ait persisté au cours des gouvernements successifs, cette volonté ne s'est jamais concrétisée. 
En effet, si les politiques fédérales et nationales argentines s'emparent de cette question en créant des dispositifs top-down (du haut vers le bas) investis par de nombreux élèves, ou bien en instituant un système parallèle, comme celui de la "gestion sociale ", elles paraissent produire des scolarités à basse intensité. À l'opposé, si en France, les ML semblent pouvoir produire des scolarités à intensités comparables à celles proposées par une partie du système traditionnel, seulement quelques milliers d'anciens décrocheurs peuvent en bénéficier, les autres étant destinés, au mieux, à un passage au sein de l'un des dispositifs d'accompagnement à l'insertion professionnelle sur lesquels l'État semble tout miser.

Ces processus en miroir permettent à l'École, respectivement française et Argentine, de perpétuer les logiques de sélection, d'exclusion et d'indifférence à la différence (Bourdieu, 1966) sur lesquelles elles se sont bâties, même au cours de leurs processus respectifs de démocratisation. Elles reproduisent ainsi la tradition du modèle de l' " inclusion excluant » (Gluz, 2015), où tout élève ne correspondant pas à l'idéal souhaité est extrait du cadre ordinaire (Muel-Dreyfus, 1975).

Au vu des fortes inégalités au sein des systèmes français et argentin, il nous semble que si ces deux sociétés ne parviennent pas à modifier leurs structures traditionnellement inégalitaires et très éloignées des principes de l' "égalité des places» (Dubet, 2010) ${ }^{28}$, alors aucune amélioration quantitative ou qualitative des dispositifs « de deuxième chance » ne pourra, à elle seule, enrayer les phénomènes d'exclusion scolaire et sociale.

\section{Bibliographie}

Acosta F. (2009), "Re-configuración de la escuela secundaria frente al ingreso masivo de jóvenes de sectores populares", Revista de Pedagogía, no 87(30), pp. 217-246.

Barrère A. (2013), "La montée des dispositifs : un nouvel âge de l'organisation scolaire ", Carrefours de l'éducation, n 36(2), pp. 95-116.

Bernstein B. (2007a), "Classe et pédagogies : visibles et invisibles ", in Deauvieau J. \& Terrail J.-P. (dir.), Les sociologues, l'école et la transmission des savoirs, Paris, La Dispute, pp. 85-112.

Bernstein B. (2007b), Pédagogie, contrôle symbolique et identité. Théorie, recherche, critique, Laval, PUL, 316 p.

28. Il s'agit, selon l'auteur, des principes de sociétés où la hiérarchisation entre les métiers n’est pas très forte. Inversement, la France et l'Argentine fonctionneraient sur les principes de l' "égalité des chances ", avec une École qui prétend offrir les mêmes chances à tous pour pouvoir exercer les métiers les plus prestigieux, dans un système où ils sont très fortement hiérarchisés. 
Bernstein B. (1997), "Écoles ouvertes, société ouverte? ", in Forquin J.-C. (dir.), Sociologues américains et britanniques, Bruxelles-Paris, De Boeck-INRP, pp. 155-164.

Bonnéry S. (2009), « Scénarisation des dispositifs pédagogiques et inégalités d'apprentissage ", Revue française de pédagogie, n 167, pp. 13-23.

Bourdieu P. (1966), "L'école conservatrice. Les inégalités devant l'école et devant la culture ", Revue française de sociologie, $n^{\circ} 7(3)$, pp. 325-347.

Cros F. (2001), Linnovation scolaire, Lyon, INRP, 124 p.

Dubet F. (2010), Les places et les chances : repenser la justice sociale, Paris, Seuil, 119 p.

Dubet F. (2002), Le déclin de l'institution, Paris, Seuil, 432 p.

Dubet F., Duru-Bellat M. \& Véretout A. (2010), Les sociétés et leur école. Emprise du diplôme et cohésion sociale, Paris, Seuil, 211 p.

Dussel I. (2004), "Inclusión y exclusión en la escuela moderna argentina: una perspectiva postestructuralist", Cadernos de Pesquisa, n 122(34), pp. 305-335.

Ebersold S. (2009), "Inclusion ", Recherche et Formation, n 61, pp. 71-83.

Giraud O. (2012), "Les défis de la comparaison à l'âge de la globalisation : pour une approche centrée sur les cas les plus différents, inspirée de Clifford Geertz ", Critique internationale, $\mathrm{n}^{\circ} 4(57)$, pp. 89-110.

Gluz N. (2015), "Jóvenes, Asignación universal por hijo y escuela secundaria: sinergias y desencuentros entre política social y escolar en Argentina", Revista Latinoamericana de Políticas y Administración de la Educación, n² 2, pp. 47-58.

Kessler G. (2006), Sociología del delito amateur, Buenos Aires, Paidós, 295 p.

Melin V. (2017), «Les structures de retour à l'école. Institutions ou dispositifs ? , Diversité, $\mathrm{n}^{\circ} 190$, pp. 43-48.

Muel-Dreyfus F. (1975), "L'école obligatoire et l'invention de l'enfance anormale », Actes de la recherche en sciences sociales, $\mathrm{n}^{\circ} 1(1)$, pp. 60-74.

Novoa A. N. \& Yariv-Mashal T. (2003), "Comparative research in education : A mode of governance or a historical journey?", Comparative Education, n 39(4), pp. 423-448.

Novoa A. (2001), "Etats des lieux de l'éducation comparée, paradigmes, avancées et impasses ", in Sirota R. (dir.), Autour du comparatisme en éducation, Paris, PUF, pp. 41-68.

Pirone F. (2015), " "Les intermédiaires scolaires". Micro-lycées et nouvelles frontières ", in Rayou P. (dir.), Aux frontières de l'école. Institutions, acteurs, objets, Saint-Denis, PUV, pp. 133-154. 
Pirone F. \& Rayou P. (2012), « Nouveaux internes, anciens décrocheurs : de l'évolution de la forme scolaire ", Revue française de pédagogie, n 179, pp. 49-62.

Poggi M. (dir) (2009), Abandono escolar y politicas de inclusión en la educación secundaria, Buenos Aires, PNUD, 206 p.

Rey B. (2014), La notion de compétence en éducation et formation. Enjeux et problèmes, Bruxelles, de Boeck, 112 p.

Rochex J.-Y. (2010), «Les "trois âges” des politiques d'éducation prioritaire : une convergence européenn? ", in Ben Ayed C. (dir.), L'école démocratique. Vers un renoncement politique?, Paris, A. Colin, pp. 94-108.

Rochex J.-Y., Southwell M. \& Indarramendi C. (dir.) (2018), Approches comparées des politiques de réduction des inégalités éducatives entre la France et l'Argentine. Nouveaux enjeux pour l'étude des inégalités scolaires et pédagogiques, rapport scientifique Action ECOS-Sud, A14HO3, Université Paris 8 Saint-Denis (France), FLACSO Buenos Aires (Argentine).

Smeyers P. \& Depaepe M. (dir.) (2009), Educational Research: The Educationalization of Social Problems, Berlin, Springer Science \& Business Media, 247 p.

Taylor C. (1992), Multiculturalism and the Politics of Recognition, Princeton University Press, Princeton, $13 \mathrm{p}$.

Terigi F. (2013), "La educación secundaria obligatoria en la Argentina: entre la expansión del modelo tradicional y las alternativas de baja escala”, Revista del IICE, $\mathrm{n}^{\circ} 33$, pp. 27-46.

Tiramonti G. (dir., 2011), Variaciones sobre la forma escolar. Limites y posibilidades de la escuela media, Homo Sapiens Ediciones, Rosario, 256 p.

Torres Molina J. (2012), "Los bachilleratos populares: una nueva educación para nuevos actores sociales”, Question, n 34(1), pp. 60-67.

Vanella L. \& Maldonado M. (dir.) (2013), Programa de Inclusión y Terminalidad de la Escuela Secundaria para Jóvenes de 14 a 17 años (PIT), Buenos Aires, UNICEF Argentina y Universidad Nacional de Córdoba, 212 p.

Zaffran J. \& Vollet J. (2016), « Comment faire pour refaire ? Les décrocheurs scolaires qui raccrochent ", Education \& Formations, n 90, pp. 137-166. 